\title{
Anatomy of sartorius muscle
}

\author{
D. Dziedzic, U. Bogacka, B. Ciszek \\ Department of Descriptive and Clinical Anatomy, Centre of Biostructure Research, Medical University of Warsaw, Poland
}

[Received 12 September 2013; Accepted 28 November 2013]

\begin{abstract}
A sartorius muscle is the longest muscle of the human system. It runs over 2 joints - hip and knee joints. In the study 10 sartorius muscles were examined. They were dissected free of lower human limbs. Dimensions of limbs which these muscles come from and dimensions of the muscles and their component parts were examined. The attention was paid mainly to parts of tendon located inside the muscle belly. The results show that they are either of a comparable length (distal tendon) or several times longer (proximal tendon) than visible parts located outside of the muscle.

Moreover, a complex structure of the distal tendon which includes 2 tracts of different places of insertion was stated. Inferior tract inserted in the same place as muscle tendons: semitendinosus and gracilis. The superior tract inserted transversely against the former one. The tendon of the sartorius muscle forms additionally an aponeurosis whose fibres enter into the deep fascia of crus. The muscle belly is characterised with various width on different levels of its length. In half of cases sword-like distal segment of belly is formed. (Folia Morphol 2014; 73, 3: 359-362)
\end{abstract}

Key words: sartorius muscle, thigh, locomotor system, pes anserinus, knee, hamstring muscles

\section{INTRODUCTION}

The sartorius muscle is the longest muscle in humans. Its name derives from a Latin word sartor - a tailor.

The sartorius muscle is situated superficially, moreover, it is distinguished by an original shape and a course. It has got a transverse section in the shape of a triangle with the base upturned. An exceptional length of this muscle often exceeds $50 \mathrm{~cm}[3,15]$.

In such long muscles not all muscle fibres run through the whole length of the muscle belly. It is estimated that at the most $30-50 \%$ of fibres run from tendon to tendon. The rest of them end intrafascicularly. The length of single fibre is estimated at 35-45 cm [5, 9, 10].

The proximal tendon of the sartorius arises from the anterior superior iliac spine. The muscle belly like an S-shaped tape twists around the anterior, and then around the medial surface of the thigh. The belly of the sartorius constitutes anterior wall of the adductor canal. Then the belly turns slantwise forward at the medial epicondyle of femur which together with quadriceps - its medial head - serves as a "trochlea" for the sartorius muscle. At the distal attachment they go into a flat divergent tendon creating in its further section superficial part of the pes anserinus [2, 4, 14].

It is worth mentioning that there are slight adaptive ethnic differences in width and the range of muscle belly and tendon of the sartorius muscle in people [11].

The described muscle receives the innervation from the femoral nerve. The vascular supply comes from the femoral artery and its branches. With respect to the innervation and vascularity, sartorius muscle has a segmental structure $[19,21]$.

The sartorius muscle is a biarticular muscle. It is the only muscle of the thigh which bends both hip joint and knee joint. It initialises the movement of

Address for correspondence: Dr D. Dziedzic, Department of Descriptive and Clinical Anatomy, Centre of Biostructure Research, Medical University of Warsaw, ul. Chałubińskiego 5, 02-004 Warszawa, Poland, tel: +48 22629 52 83, fax: +48 22629 52 83, e-mail: policeros@wp.eu 
flexion in both joints from the phase of full extension, while other flexors need its initial flexion as an initial position to begin a movement [8].

The sartorius muscle is a weak external rotator and a weak abductor of the hip joint. Having an effect on a crus, it rotates it internally while a knee joint is flexed $[7,18]$.

This muscle plays an important part in stabilisation of pelvis, especially in women. It happens thanks to constrictive effect which both muscles, right and left, have on pubic symphysis [13].

Injuries of the sartorius muscle most often occur in the area of proximal and distal attachment. In young people, anterior superior iliac spine avulsion, caused by common activity of the sartorius muscle and tensor fasciae latae, may be observed. In the area of pes anserinus different pathologies connected with frequently repetitive movements leading to micro injuries and tissue loads in this area are met. In the result of this, inflammations of bursa anserina, tendon strains and rupture may take place. Such injuries happen to: i.a. athletes, runners, jumpers and football players $[1,15$, $16,20]$. During harvesting of the hamstring tendons for the cruciate ligament reconstruction, always the distal tendon of the sartorius is partly injured.

The summarised above knowledge on the external anatomy of the sartorius muscle is quite extensive. The information on the morphometry and inner structure of the muscle are limited so the present study was undertaken.

\section{MATERIALS AND METHODS}

Research were carried out on a ten lower limbs (8 left and 2 right) fixed in formalin, which was in the Department of Descriptive and Clinical Anatomy, Biostructure Centre of Medical University of Warsaw. A preparation method was used to separate superficial tissues reaching the muscle. Then, after preparation of fascia, the sartorius muscle was examined through the whole length. A morphological structure of tendons, both free parts and parts located inside the muscle belly, was measured and described.

The following measurements were done:

- absolute length of the limb - measured from anterior superior iliac spine to medial ankle;

- relative length of the limb - measured from greater trochanter to medial ankle;

- absolute length of thigh - measured from anterior superior iliac spine to medial aspect of knee joint gap;
- relative length of thigh - measured from greater trochanter to lateral aspect of knee articular space;

- length of the whole muscle with tendons - from proximal to distal attachment;

- length of muscle belly - measured from the beginning of first muscle fibres to the end of the last ones;

- lengths of the whole proximal and distal tendon measured from their attachments to maximum range of tendinous fibres inside the muscle;

- lengths of the parts of proximal and distal tendon inside the muscle belly and outside the muscle belly (free part);

- width of the muscle at $1 / 4,1 / 2,3 / 4$ and in the widest place at the distal end.

\section{RESULTS}

The proximal tendon of sartorius muscle goes from the anterior superior iliac spine. After $0.5-2 \mathrm{~cm}$ of free course it enters the muscle. The shape of the tendon part located beyond the muscle belly resembles a trapeze with the base upturned. The part of the tendon hidden in the muscle belly is longer several times than the former one (Fig. 1). In examined preparations its length was within $4-7 \mathrm{~cm}$.

A long muscle belly of the sartorius muscle (45$-58 \mathrm{~cm}$ ) arranges itself in the S-shape in the thigh. In most cases $(9 / 10)$ the initial part of the muscle was characterised by the shortest width $(2.2-3.8 \mathrm{~cm})$.

The widest part of the muscle $(2.6-4.4 \mathrm{~cm})$ was located variably: in 1 case it was the proximal part of the belly, in 3 cases - middle, in 5 cases - distal, in 1 case medial as well as distal (the same dimension, bigger than the proximal part). In 6 out of 10 cases described in the study the part of the muscle located by the medial condyle was characterised by sword-like extension (Fig. 2) which was situated on the medial side of the muscle belly and enlarged the width of this part of the muscle of $0.2 \mathrm{~cm}$ to $1 \mathrm{~cm}$.

The specific anatomical structure of the distal tendon $(13-17 \mathrm{~cm})$ of the sartorius muscle was stated. Similar to the proximal tendon, it has its extramuscular $(4-10.5 \mathrm{~cm})$ and intramuscular part $(6-11 \mathrm{~cm})$. In 1 case lengths were the same $(8 \mathrm{~cm})$, in 6 cases the part hidden in the muscular belly was longer, in the last 3 cases the free part was the longer.

The anatomical structure of the distal part of the tendon was constant. Its fibres approaching to the terminal attachment arrange themselves in 2 tracts 


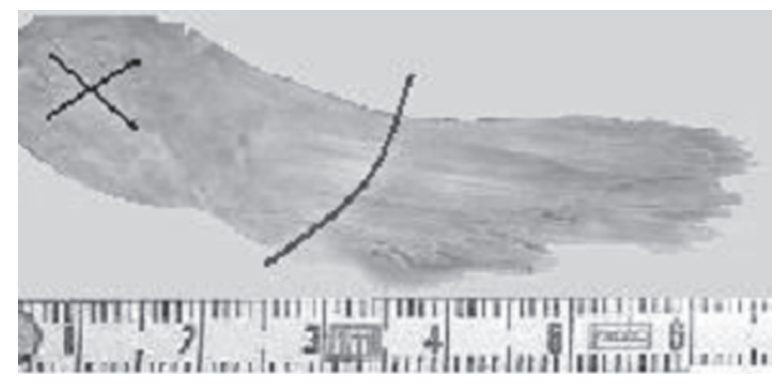

Figure 1. Proximal tendon of sartorius muscle: intra- and extramuscular part between borderline; $\mathrm{x}$ - anterior superior iliac spine.

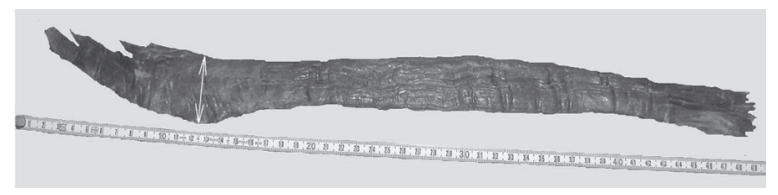

Figure 2. Muscle belly of sartorius muscle with sword-like extension of distal part.

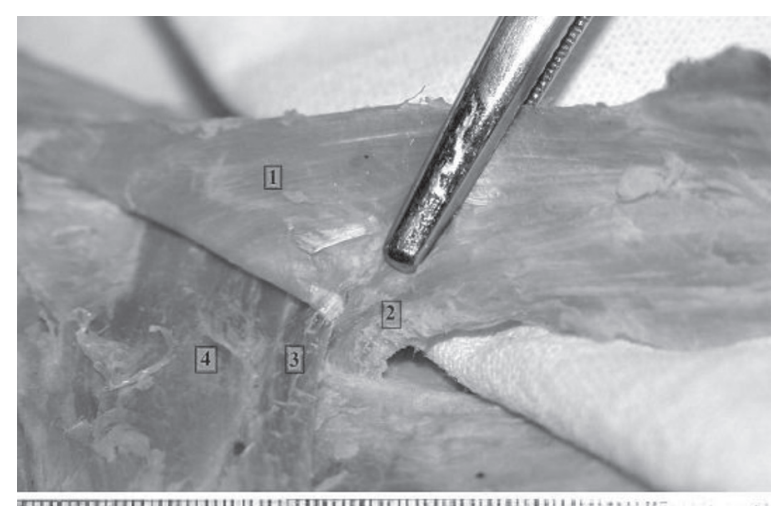

Figure 3. End of sartorius distal tendon: inferior tract (1) and superior tract (2). Tendon of gracilis (3) and semitendinosus muscles (4).

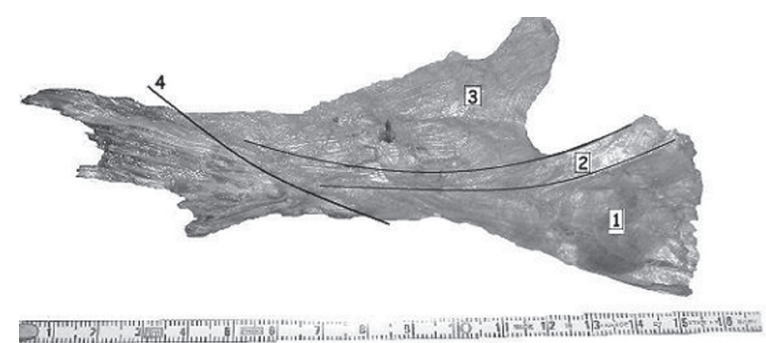

Figure 4. Distal tendon of sartorius muscle; 1 - inferior tract; 2 - superior tract; 3 - aponeurosis; 4 - borderline between intra- and extramuscular part.

and aponeurosis. Each of them ends in different place. Inferior tract covers insertion of remaining mu- scles of pes anserinus - gracilis and semitendinosus. Its fibres diverging like in the range reaching the common insertion with muscles mentioned before. The place of the insertion has a shape of a vertical (Figs. 3, 4), slightly rounded section located below the tibial tuberosity.

Superior tract strand is more cohesive than the inferior tract. It can be easily separated from it. Its fibres are shorter because they end at the upper edge of gracilis tendon. The place of the insertion, as opposed to inferior tract, is thus situated transversely in relation to the tibia - below and medially from the tibial tuberosity. The fibres go under the upper edge of terminal part of gracilis tendon which covers the insertion of this tract (Figs. 3, 4).

Aponeurosis of the sartorius muscle is one accessory band that arises from the upper part of the distal tendon. It is a flat bundle of fibres located medially in relation to the tibial tuberosity. It runs on its height and in the direction of it. The fibres diverge and they reach their end entering into the deep fascia of crus (Fig. 4).

\section{DISCUSSION}

Data concerning initial attachment and muscle length were confirmed. Data concerning terminal attachment, morphology of both tendons and muscle belly present new features of the sartorius muscle. Similar result were not found in available literature $[2,3,12]$.

We have measured not only the free part of the tendon, located beyond the muscle belly, but also parts of these tendons located inside the muscle belly. This kind of research procedures were not used by other authors [2, 3, 6, 12]. Knowledge on intramuscular course of the tendon is important during ultrasonography or magnetic resonance imaging and evaluation of the muscle-tendon injuries.

The length of the muscle is described in literature as over $50 \mathrm{~cm}[6,10]$. However, not all authors explained if the muscle was measured with its tendons "from bone to bone" or only the belly was measured. In the present study it was stated that average length of muscle measured from the initial attachment to the terminal one was $57.5 \mathrm{~cm}$, and the average length of the muscle belly $-49.2 \mathrm{~cm}$.

There is similar situation with the description of length of tendons. Data from literature estimate that it equals about $8 \mathrm{~cm}$ without clear marking which 
part of the tendon was measured and which tendon proximal or distal was evaluated [9]. Referring these data to the present study, they can be attributed to the length of the terminal tendon, precisely to its part which is outside the muscle belly, whose average lenght amounts $7.0 \mathrm{~cm}$.

Morphometric data relating to the parts of both tendons inside the muscle belly were not found in available literature $[2,3,12]$.

In the case of the distal tendon, the average length of the part inside the muscle belly equals $8.5 \mathrm{~cm}$. In the proximal tendon, the length of its intramuscular part exceeds the length of free part several times. The average length of the free part equals $1.3 \mathrm{~cm}$, while the average of the length of the tendon hidden in the muscle belly is $5.35 \mathrm{~cm}$.

The shape of the muscle belly in many cases (in half of the examined cases), approaching the distal tendon exceeds its transverse diameter, creating the typical extension in the shape of the sword. In available literature descriptions concerning this feature were not found $[2,3,12,19]$.

In all examined cases, a precisely described complex structure of the distal part of terminal tendon was never stated before $[2,3,12]$. It is divided into the superior and inferior tract of this tendon which differs in the place of attachment. The described attachment below the tibial tuberosity is the inferior tract of the distal tendon of the sartorius muscle. The most cohesive is the superior tract which attaches transversely in relation to the previous one creating an angle near right with it.

The upper part of the further section of the distal tendon creates aponeurosis. It is a dispersed bundle of fibres which do not have a typical attachment to the bone structure. They end entering into the fascia of leg.

It is worth mentioning that distal end of the sartorius muscle in mammals is described as the aponeurosis emitting its fibres in the medial part of the upper part of crus [17]. This fact can explain the identification of the aponeurosis of the sartorius muscle in the present study in humans.

\section{CONCLUSIONS}

This complexity of the distal tendon is important during open surgery for the pes anserinus region and medial ligamentous complex of the knee as well during harvesting the hamstring tendons. The sartorius aponeurosis covers the tibial collateral ligament. Fibres of this aponeurosis enters the system of the medial patellar retinacula according to the surgical point of view.

\section{REFERENCES}

1. Bencardino JT, Rosenberg ZS, Brown RR, Hassankhani A, Lustrin ES, Beltran J (2000) Traumatic musculotendinous injuries of the knee: diagnosis with MR imaging. Radio Graphics, 20: 103-120.

2. Burnet NG, Bennet-Briton $T$, Hoole AC, Jefferies S J, Parkin IG (2004) The anatomy of sartorius muscle and its implications for sarcoma radiotherapy. Sarcoma, 8: 7-12.

3. Clavert P, Cognet JM, Baley S, Stussi D, Prevost P, Babin SR, Simon P, Kahn JL (2008) Anatomical basis for distal sartorius muscle flap for reconstructive surgery below the knee. Anatomical study and case report. J Plastic, Reconstr Aesthetic Surg, 61: 50-54.

4. Duda GN, Brand D, Freitag S, Lierse W, Schneider E (1996) Variablity of femoral muscle attachments. J Biomech, 29; 1185-1190.

5. Harris AJ, Duxon M, Butler JM, Hodges PW, Taylor JL, Gandevia SC (2005) Muscle fiber and motor unit behavior in the longest human skeletal muscle. J Neurosci, 14: 8528-8533.

6. Heron M, Richmond F Jr. (1993) In-series fiber architecture in long human muscles. J Morphol, 216: 35-45.

7. Hoek van Dijke G, Snijders C (1999) A biomechanical model on muscle forces in the transfer of spinal load to the pelvis and legs. J Biomech, 33: 927-933.

8. Johnson CE, Basmajian JW, Dasher W (1972) Electromyography of sartorius muscle. Anat Rec, 2: 127-130.

9. Klein Horsman M, Koopman H (2007) Morphological muscle and joint parameters for musculoskeletal modelling of the lower extremity. Clin Biomech, 22: 239-247.

10. Lieber $R$ (2002) The phisiological basis of rehabilitation. Lippincott Williams and Wilkins, Philadelphia.

11. Loth E (1934) Anthropologie des parties molles. Masson \& Cie, Paris.

12. Mathers LH Jr. Chase A, Dolph J, Glasgow EF, Gosling JA (1996) Clinical anatomy. Mosby-Year Book, St Louis.

13. Meyers W, Greenleaf R (2000) Anatomic basis for evaluation of abdominal and groin pain in athletes. Operative Tech Sports Med, 13: 55-61.

14. Mochizuki T, Akita K (2004) Pes Anserinus: layered supportive structure on the medial side of the knee. Clin Anat, 17: 50-54.

15. Morelli V, Smith V (2001) Groin injuries in athletes. Am Family Physician, 64: 1405-1414.

16. Pointinger H, Munk P, Poeschl GP (1999) Ruptur des Pes anserinus superficialis und Teilruptur des Lig. Patellae, Unfallchirurg, 102: 69-73.

17. Poplewski R (1948) Anatomia ssaków. Vol. 3. Czytelnik, Stockholm.

18. Pressel T, Lengsfeld M (1998) Functions of hip joint muscles. Medical Engineering and Physics, Philadelphia.

19. Tanaka C, Ritomy IM, Rodrigues AJ Jr. (2006) Anatomical contribution to the surgical construction of the sartorius muscle flap. Surgi Radiol Anat, 28: 277-283.

20. White KK, Williams SK, Mubarak SJ (2002) Definition of two types of anterior superior iliac spine avulsion fractures. J Pediatric Orthopaedics, 22: 578-582.

21. Wysocki J, Krasuski P, Czubalski A (1996) Vascularization of the sartorius muscle. Folia Morphol, 55: 115-120. 